\title{
Low energy deuteron elastic scattering on light and medium nuclei
}

\author{
M. Avrigeanu ${ }^{1, a}$, H. Leeb ${ }^{2}$, W. von Oertzen ${ }^{3}$, F.L. Roman ${ }^{1}$, and V. Avrigeanu ${ }^{1}$ \\ 1 EURATOM-MEdC Fusion Association, "Horia Hulubei” National Institute for Physics and Nuclear Engineering, P.O. Box MG-6, \\ 76900 Bucharest, Romania \\ 2 Atominstitut der Osterreichischen Universitaeten, Technische Universitaet Wien, Wien, Austria \\ ${ }^{3}$ Freie Universität Berlin, Fachbereich Physik, 14195 Berlin, and Hahn-Meitner-Institut, 14109 Berlin, Germany
}

\begin{abstract}
An analysis of the elastic scattering of deuterons on ${ }^{6,7} \mathrm{Li},{ }^{27} \mathrm{Al},{ }^{54,56,58} \mathrm{Fe},{ }^{63,65} \mathrm{Cu}$, and ${ }^{93} \mathrm{Nb}$ for energies from 3 to $60 \mathrm{MeV}$ has been carried out using a semi-microscopic optical potential which consists of a Coulomb term, a real double-folding (DF) potential, a phenomenological imaginary potential and a spin-orbit component. No normalization constant was involved in this work for the DF real potential, in order to emphasize the effects of further corrections requested by a satisfactory description of the experimental data. Moreover, an empirical non-locality correction to the microscopic real potential has been added in the case of the $\mathrm{d}+{ }^{6} \mathrm{Li}$ scattering. By taking into account also the dispersion correction, a good agreement of the experimental and semi-microscopic elastic scattering angular distribution has been obtained. The imaginary and spin-orbit potential parameters obtained by the semimicroscopic data analysis have been kept fixed within a second step of the present analysis, in order to obtain a full phenomenological optical potential needed for applications. As a global result, one may note differences of about $10-15 \%$ between the deuteron reaction cross sections corresponding to the present improved optical potential and those obtained using the default input parameters within the TALYS code, while enlarged changes are shown by the calculated elastic-scattering angular distributions. The differences with respect to the evaluated total reaction cross sections of the ACSELAM library are about $25-30 \%$.
\end{abstract}

\section{Introduction}

The development of novel nuclear technologies (e.g., power plants based on nuclear fusion) requires a quantitative knowledge of all relevant nuclear reactions. In fusion facilities the effective interactions of deuterons with light nuclei as well as structural materials are of particular interest. Thus, calculations of the D-Li source term [1,2] as well as deuteron induced activation cross sections for structural materials such as $\mathrm{Al}, \mathrm{Fe}, \mathrm{Cu}$ and $\mathrm{Nb}[3]$ are important and have been performed. At present, taking into account the requests of fusion research, there is a significant effort to develop dedicated databases for fusion applications [4]. Recently, specific aspects with regard to fusion neutronics have been taken into account [5], e.g. in the activation cross section library ACSELAM [6] and the computer code TALYS [7].

Concerning the deuterons, unlike in the nucleon case, there are no global optical model potential (OMP) which describe the scattering data over a wide range of nuclei and energies sufficiently well (see, e.g., the IAEA Reference Input Parameter Library [8]). This fact leads to an increased uncertainty in calculations of deuteron-induced reaction cross sections because the optical model is the basic ingredient of almost all nuclear model calculations. Moreover, the weak binding of the deuteron results in significant contributions of the breakup channel and enhances a variety of reactions at low bombarding energy. Nevertheless, a semi-microscopic analysis [1] appears as an appropriate basis for the evaluation of the deuteron-nucleus optical potential. These potentials may be used for calculations of the deuteron-induced activation cross sections required for fusion applications.

\footnotetext{
${ }^{a}$ Presenting author, e-mail: mavrig@ifin.nipne.ro
}

\section{Semi-microscopic analysis of deuteron elastic scattering}

The deuteron elastic-scattering experiments on $\mathrm{Al}, \mathrm{Fe}, \mathrm{Cu}$ and $\mathrm{Nb}$ isotopes, at incident energies lower than $20 \mathrm{MeV}$, are not satisfactorily described by any of the phenomenological well-known OMPs of either Perey and Perey [9], Lohr and Haeberli [10], Daehnick et al. [11], or Bojowald et al. [12] as well as the recent analyses [13]. Moreover, the analysis of the low-energy elastic scattering data suffers from discrete and continuous ambiguities in the OMP parameters, whose uncertainties vary for various target nuclei and for different incident energies due to the precision of data analyzed. Thus, in order to reduce the phenomenological character of previous analyses [1,2], we carried out an extended analysis using a semi-microscopic optical potential based on a real double-folding (DF) potential (e.g., [14] and refs. therein) and phenomenolgical imaginary and spin-orbits terms. The parameters of the latter are determined by a fit of elastic-scattering differential and reaction cross sections of deuterons on ${ }^{27} \mathrm{Al},{ }^{54,56,58, \text { nat }} \mathrm{Fe},{ }^{63,65 \text {, nat }} \mathrm{Cu}$ and ${ }^{93} \mathrm{Nb}$ (table 1) for energies from 3 to $60 \mathrm{MeV}$ [15]. It should be emphasized that no adjustable parameter or normalization constant was involved for the real potential, in order to couple the imaginary and spin-orbit part so that the predictive power of the semi-microscopic potential is preserved. As a first result, this approach may reduce the number of the OMP parameters as well as the corresponding uncertainties.

The direct and exchange components of the real central potential are given in terms of the projectile and target nuclear densities, which are folded with the Paris M3Y effective NN interaction [16]. The nuclear density 
Table 1. The experimental data [15] of elastic-scattering angular distributions and total reaction cross sections analyzed in this work.

\begin{tabular}{ccc}
\hline Target & \multicolumn{2}{c}{$E_{d}(\mathrm{MeV})$} \\
\cline { 2 - 3 } & $d \sigma / d \vartheta$ & $\sigma_{R}$ \\
\hline${ }^{6} \mathrm{Li}$ & $3,4,5,6,7,8,9,10,11.8,12,14.7$, \\
& $19.6,50$ \\
${ }^{7} \mathrm{Li}$ & $3,4,5,6,7,8,9,10,11.8,12,14.7$ \\
${ }^{27} \mathrm{Al}$ & $5,7,9,9.8,11,11.4,11.8,12.8,13,15$, & $13.4,24.9$ \\
& $52,58.7$ & 12.3 \\
${ }^{54} \mathrm{Fe}$ & $10,12,12.3,13,14.5,34.4,52,56$ \\
${ }^{56} \mathrm{Fe}$ & $5,7,11.8,12,12.3,30,56$ & 12.3 \\
${ }^{58} \mathrm{Fe}$ & 12.3 & 12.3 \\
${ }^{n a t} \mathrm{Fe}$ & $11.8,15,25.9$ & $13.4,25.1$ \\
${ }^{63} \mathrm{Cu}$ & $12,14.5,34.4$ & $11.93,13.31$ \\
${ }^{65} \mathrm{Cu}$ & $12,34,4$ & $11,24.4$ \\
${ }^{n a t} \mathrm{Cu}$ & $11.8,15,21.6$ & \\
${ }^{93} \mathrm{Nb}$ & $11.8,15,34.4,52$ & \\
\hline
\end{tabular}

distributions of the target nuclei have been described by Gaussian or Fermi distributions with the parameters provided by the electron scattering data analysis [17]. The deuteron density distribution has been obtained both from the wave function calculated with the Paris as well as CD-Bonn potentials [18], and from the experimental charge form factors [19]. Moreover, since a true microscopic treatment leads to a non-local optical potential, we have to include also a non-locality correction to the DF real potential [2] because we must use equivalent local potentials (ELP) within the available standard codes. The Fiedeldey et al. [20] correction has been adopted in this respect in the case of the $\mathrm{d}+{ }^{6} \mathrm{Li}$ scattering, which arises from the dynamical coupling of the elastic to non-elastic channels, and may be important in the processes involving clustered nuclei mainly due to their great probability of break-up. Finally we have also taken into account the dispersion correction to the real potential provided by the phenomenological imaginary potential part, by means of the analytical solution and linear schematic model of Mahaux et al. [21]. The average energy dependence of the surface potential depths has been involved in this respect.

\section{Average energy-dependent phenomenological OMP}

The differential elastic-scattering cross sections have been calculated by a modified version of the code SCAT2 [22] which includes the DF model results as an option for the OMP real part. Within the first step of the present work we looked for the imaginary and spin-orbit potential parameters able to describe, together with the microscopic real potential, the elasticscattering differential cross sections. The results for ${ }^{27} \mathrm{Al}$ from 5 up to $60 \mathrm{MeV}$ are shown in figure 1 (left side). Next, the imaginary and spin-orbit potential parameters obtained by the semi-microscopic data analysis have been kept fixed within a second step of the present analysis, in order to obtain a full phenomenological OMP needed for applications. The advantage of having well settled already at least half of the usual OMP parameters increases obviously the effectiveness of
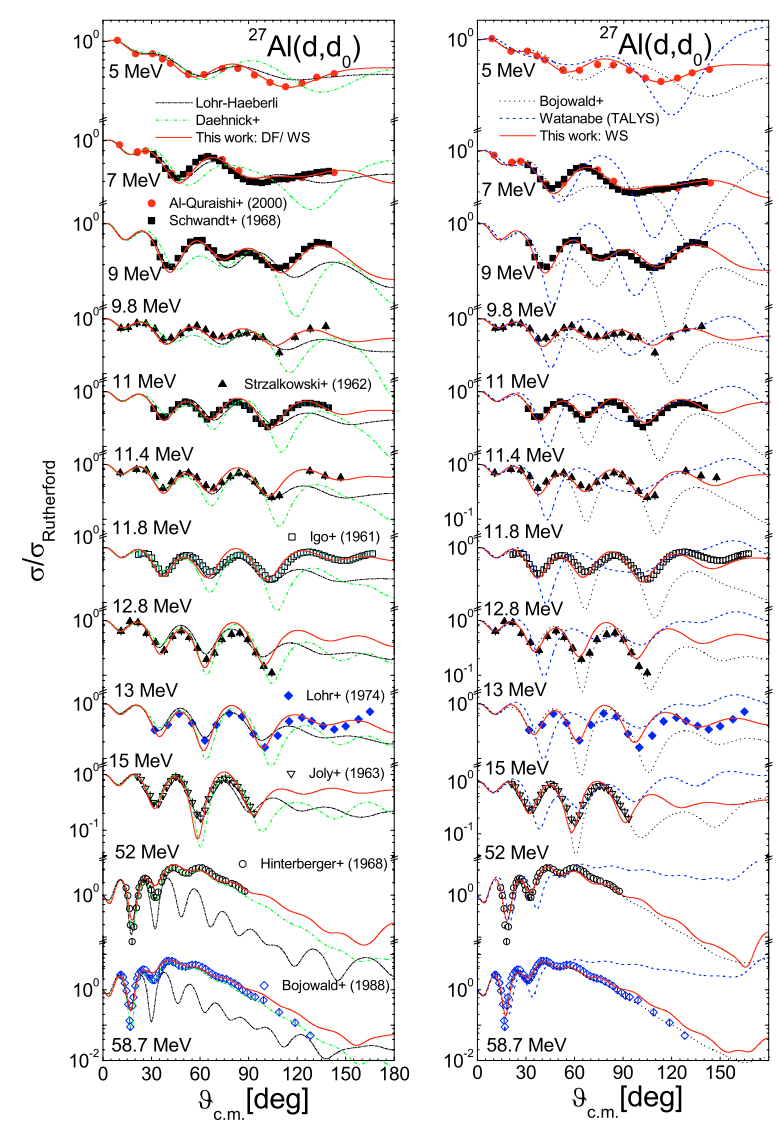

Fig. 1. Comparison of experimental [15] and calculated angular distributions of the elastic scattering of deuterons on ${ }^{27} \mathrm{Al}$ between 5 and $60 \mathrm{MeV}$ using the present semi-microscopic (solid curves, left side) and phenomenological (right side) OMPs, as well as the global potentials [10] (dashed curves), [11] (dash-dotted curves), and [12] (dash-dot-dotted curves).

fitting the data. Based on the corresponding local OMP parameters, the average energy-dependent OMP parameters have been obtained for each element. The corresponding elasticscattering differential cross sections are shown in figures 1-3.

In conclusion, an improved description of the experimental data [15] from 5 to $60 \mathrm{MeV}$ is provided with respect to the predictions of the above-mentioned global OMP parameter sets [10-12] and the default OMP within the code TALYS [7]. The latter makes use of the nucleon OMP of Koning and Delaroche [24] and applies a simplification of the folding approach of Watanabe [23]. The same comparison was performed for deuteron reaction cross sections (fig. 4), considering also the evaluated data of the ACSELAM library [6].

Summarizing these results, one observes differences of about $10-15 \%$ between the deuteron reaction cross sections corresponding to the present improved optical potential and those obtained using the default input parameters within the code TALYS, while enlarged changes are shown by the calculated deuteron elastic-scattering angular distributions. The differences with respect to the evaluated total reaction cross sections of the ACSELAM library are about 25-30\%. An additional comparison between the experimental data and the angular distributions calculated using the deuteron global OMP of Bojowald et al. [12] showed the lack of reliability 

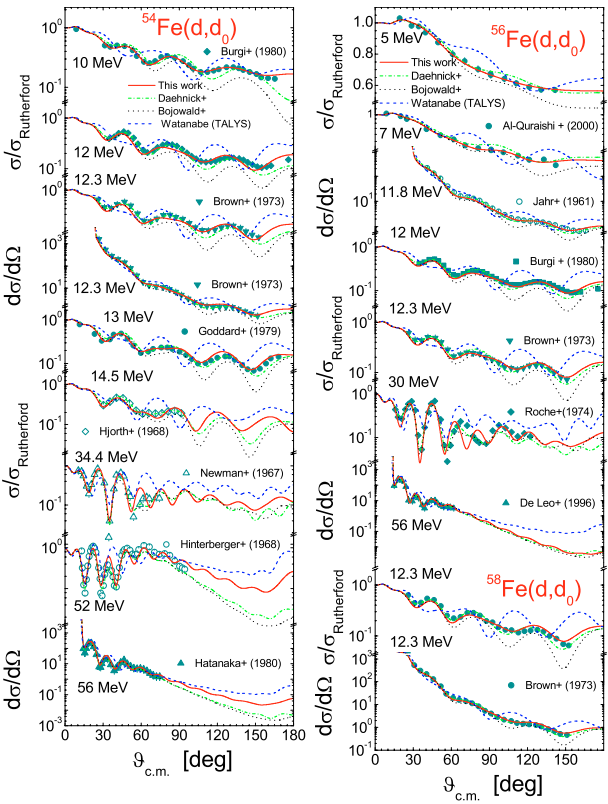

Fig. 2. Comparison of experimental [15] and calculated angular distributions of the elastic scattering of deuterons on ${ }^{54,56,58} \mathrm{Fe}$ isotopes between 5 and $56 \mathrm{MeV}$ using the present phenomenological OMP (solid curves), the global potentials [11] (dash-dotted curves) and [12] (dotted curves), and the TALYS option based on Watanabe approach (dashed curves).
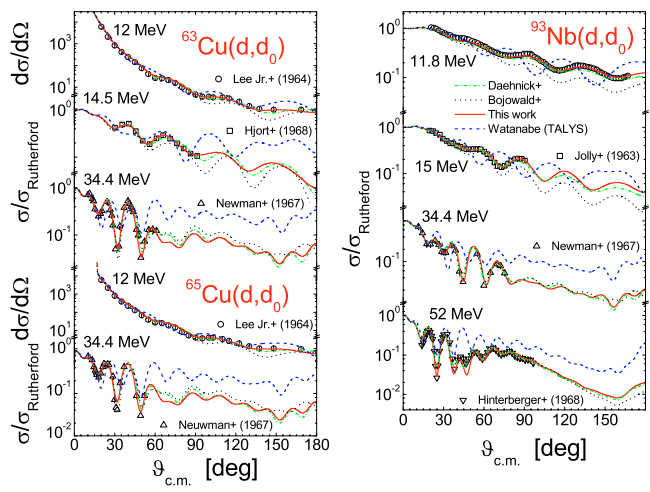

Fig. 3. Comparison of experimental [15] and calculated angular distributions of the elastic scattering of deuterons on ${ }^{63,65} \mathrm{Cu}$ and ${ }^{93} \mathrm{Nb}$ isotopes between 12 and $52 \mathrm{MeV}$ using the present phenomenological OMP (solid curves), the global potentials [11] (dash-dotted curves) and [12] (dotted curves), and the TALYS option based on Watanabe approach (dashed curves).

of this OMP at energies lower than $25 \mathrm{MeV}$, i.e. well below the energy range for which it was established, while it is well suited at energies about $50 \mathrm{MeV}$.

\section{Effects on the activation cross-section evaluation}

Finally, the average energy-dependent phenomenological OMPs obtained in the present work have been used for calculations of activation cross-section for deuterons incident on ${ }^{27} \mathrm{Al},{ }^{54,56} \mathrm{Fe},{ }^{63,65} \mathrm{Cu}$ and ${ }^{93} \mathrm{Nb}$ nuclei [14]. The computer

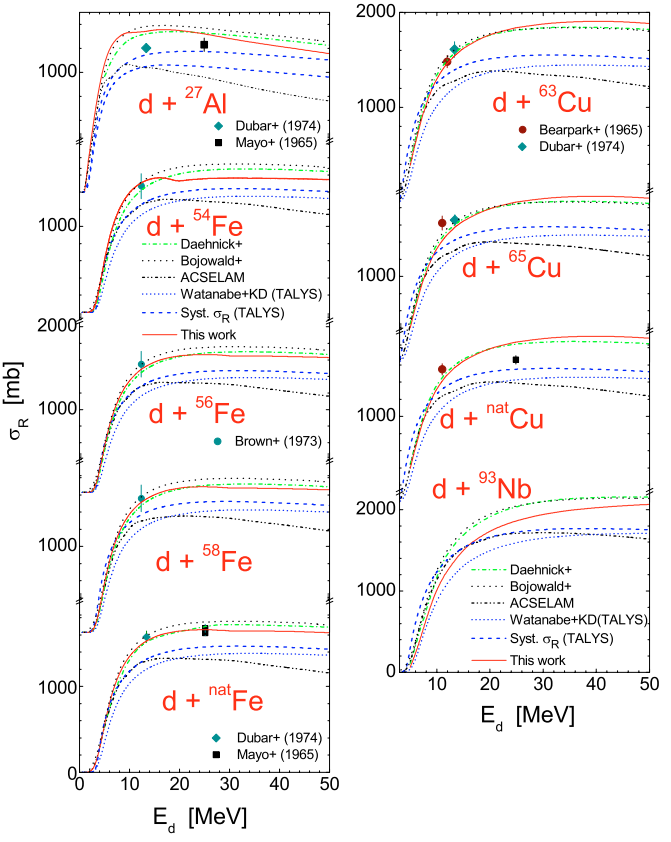

Fig. 4. Comparison of experimental [15] and calculated total reaction cross sections of deuterons on ${ }^{27} \mathrm{Al},{ }^{54,56,58, \text { nat }} \mathrm{Fe},{ }^{63,65, \text { nat }} \mathrm{Cu}$ and ${ }^{93} \mathrm{Nb}$ up to $50 \mathrm{MeV}$, by using the present average energydependent phenomenological OMP (solid curves) as well as the global potentials [11] (dash-dotted curves), [12] (dotted curves), the TALYS options based on Watanabe approach (short-dashed curves) and systematics of non-elastic cross sections (dashed curves), and the ACSELAM library [6] (dash-dot-dotted curves).

codes TALYS and EMPIRE-II [25] have been applied with default parameters except OMPs for deuterons which have been replaced by the present phenomenological one.

A sample case is shown in figure 5 for the ${ }^{93} \mathrm{Nb}(\mathrm{d}, 2 \mathrm{n})^{93 \mathrm{~m}} \mathrm{Mo}$ reaction, by taking the opportunity of rather recent experimental data. One observes a satisfactory agreement between the measured and calculated cross sections obtained by various potential at incident energies which are quite close to the reaction threshold. Despite the achieved improvements, the situation is still not fully satisfactory as can be seen from the large differences in the deuteron-induced reaction cross sections (fig. 4) and the elastic-scattering differential cross sections (fig. 3). The actual databases should be improved to allow for more reliable model validation. On the other hand it could be pointed out the lack of possibility for further trial of the ACSELAM library by means of the experimental cross sections for isomeric states. This follows the use of the pre-equilibrium emission (PE) and statistical-model computer code ALICE for the production of the corresponding evaluated data [6], quite powerful by inclusion of the PE parameter-free Geometry Dependent Hybrid model [26], but not taking into account the angular-momentum and parity conservation within either PE or equilibration processes.

The authors acknowledge the useful discussions and advices of Arjan Koning and Mike Herman on calculations performed with the codes TALYS and EMPIRE-II, respectively. Work supported in part by the European Communities EFDA under the contract of Association EURATOM/MEdC-Bucharest. 


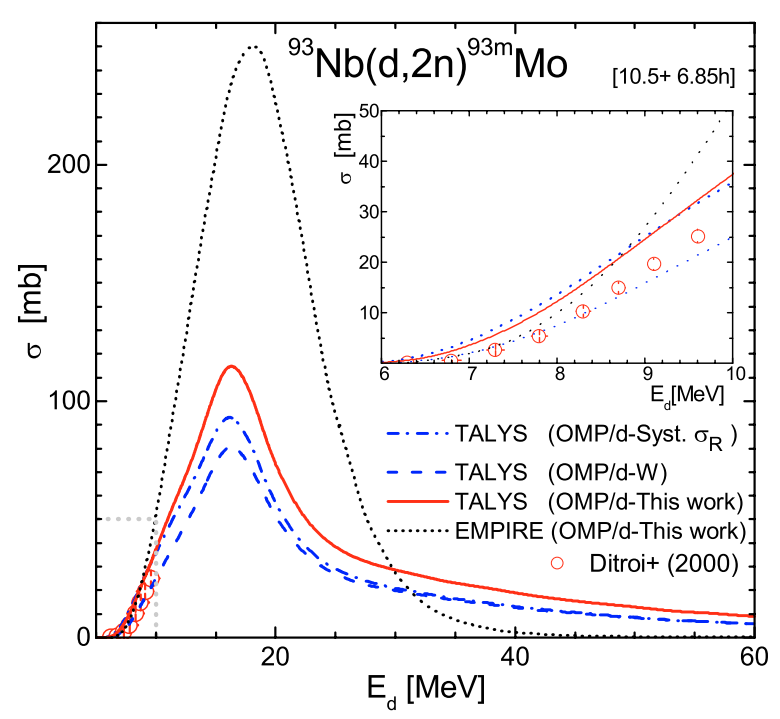

Fig. 5. Comparison of the experimental and calculated ${ }^{93} \mathrm{Nb}(\mathrm{d}, 2 \mathrm{n}){ }^{93 \mathrm{~m}}$ Mo reaction cross sections, using the codes TALYS (solid curve) and EMPIRE-II (dotted curve) with default input parameters excepting the deuteron average energy-dependent phenomenological OMP of this work, and the TALYS options based on Watanabe approach (dashed curve) and systematics of non-elastic cross sections (dash-dotted curve).

\section{References}

1. M. Avrigeanu, W. von Oertzen, U. Fischer, V. Avrigeanu, Nucl. Phys. A 759, 327 (2005).

2. M. Avrigeanu, W. von Oertzen, H. Leeb, F.L. Roman, V. Avrigeanu, in Proc. 11th Int. Conf. on Nuclear Reaction Mechanisms, 12-16 June 2006, Varenna, Italy, edited by E. Gadioli, (Ricerca Scientifica ed Educazione Permanente, Milano, 2006), p. 193.

3. M. Avrigeanu, F.L. Roman, V. Avrigeanu, in Proc. Int. Workshop on Activation Data - EAF 2007, Prague, Oct. 2-4, 2006, www.ojr.ic.cz/.

4. Y. Capouet, J. Pamela, in Workshop on a Coordination or Support Action on Fusion, Data - International Fusion Data bases, EFDA CSU, Garching, 23 Feb. 2007, http://efdasql.ipp. mpg.de/eumeetings/databases/.

5. K. Thomsen, EU Fusion Research Data Base Survey, http://efdasql.ipp.mpg.de/eumeetings/databases/References/ Brussels23feb2007/DatabaseSurvey11.ppt.
6. S. Tanaka, N. Yamano, Table of Isotope Production Cross Sections (ACSELAM Library), JAERI, 1998, http://wwwndc.tokaisc.jaea.go.jp/ftpnd/sae/acl.html.

7. A.J. Koning, S. Hilaire, M.C. Duijvestijn, Report 21297/ 04.62741/P FAI/AK/AK, NRG-Petten, 2004; A.J. Koning, M.C. Duijvestijn, Nucl. Phys. A 744, 15 (2004); http://www. talys.eu/.

8. T. Belgya, O. Bersillon, R. Capote, T. Fukahori, G. Zhigang, S. Goriely, M. Herman, A.V. Ignatyuk, S. Kailas, A. Koning, P. Oblozinsky, V. Plujko, P. Young, Handbook for calculations of nuclear reaction data, RIPL-2, IAEA-TECDOC-1506 (IAEA, Vienna, 2006), http://www-nds.iaea.org/RIPL-2/.

9. C.M. Perey, F.G. Perey, Phys. Rev. 32, 755 (1963).

10. J.M. Lohr, W. Haeberli, Nucl. Phys. A 232, 381 (1974).

11. W.W. Daehnick, J.D. Childs, Z. Vrcelj, Phys. Rev. C 21, 2253 (1980).

12. J. Bojowald, H. Machner, H. Nann, W. Oelert, M. Rogge, P. Turek, Phys. Rev. C 38, 1153 (1988).

13. P. Chau Huu-Tai, Nucl. Phys. A 773, 56 (2006).

14. M. Avrigeanu, W. von Oertzen, V. Avrigeanu, Nucl. Phys. A 764, 246 (2006).

15. Experimental Nuclear Reaction Data (EXFOR), http://wwwnds.iaea.or.at/exfor.

16. N. Anantaraman, H. Toki, G. Bertsch, Nucl. Phys. A 398, 279 (1983).

17. K.H. Bray, M. Jain, K.S. Jayaraman, G. Lobianco, G.A. Moss, et al., Nucl. Phys. A 189, 35 (1972); J.W. Negele, Phys. Rev. C 1, 1260 (1970).

18. R. Machleidt, Adv. Nucl. Phys. 19, 189 (1989); Phys. Rev. C 63, 024001 (2001).

19. D. Abbott, A. Ahmidouch, H. Anklin, J. Arvieux et al., Phys. Rev. Lett. 84, 5053 (2000).

20. H. Fiedeldey, R. Lipperheide, G.H. Rawitscher, S.A. Sofianos, Phys. Rev. C 45, 2885 (1992).

21. C. Mahaux, H. Ngo, G.R. Satchler, Nucl. Phys. A 449, 354 (1986); Nucl. Phys. A 456, 134 (1986).

22. O. Bersillon, Centre d'Études de Bruyères-le-Châtel, Note CEA-N-2227, 1992.

23. S. Watanabe, Nucl. Phys. 8, 484 (1958).

24. A.J. Koning, J.P. Delaroche, Nucl. Phys. A 713, 231 (2003).

25. M. Herman, P. Oblozinsky, R. Capote, M. Sin, A. Trkov, A. Ventura, V. Zerkin, in Proc. Int. Conf. on Nuclear Data for Science and Technology (ND2004), Santa Fe, 2004, edited by R.C. Haight, M.B. Chadwick, T. Kawano, P. Talou, (American Institute of Physics, New York, 2005), p. 1184; EMPIRE-II v.2.19, http://www-nds.iaea.org/empire/.

26. M. Blann, H.K. Vonach, Phys. Rev. C 28, 1475 (1983). 